\title{
Produtividade da cana-de-açúcar em Mato Grosso do Sul e Goiás: uma análise a partir da Visão Baseada em Recursos
}

\author{
Productivity of sugarcane in Mato Grosso do Sul and Goiás: a Resource-Based \\ View analysis
}

\section{Productividad de la caña de azúcar en Mato Grosso do Sul y Goiás: un análisis a partir de la Visión Basada en Recursos}

\author{
Roberto Bernardo ${ }^{1}$ \\ Wagner Luiz Lourenzani ${ }^{2}$ \\ Eduardo Guilherme Satolo ${ }^{2}$ \\ Marcellus Marques Caldas ${ }^{3}$
}

\begin{abstract}
Resumo: Baseado na teoria da Visão Baseada em Recursos (VBR), este trabalho analisou como os recursos internos das propriedades rurais de cana-de-açúcar nos estados de Mato Grosso do Sul e Goiás influenciam seu desempenho agrícola. Foi um estudo aplicado de caráter exploratório, envolvendo uma pesquisa de campo com 148 produtores rurais. A análise quantitativa revelou diferenças entre os padrões de produção de cana-de-açúcar nas duas regiões de estudo.
\end{abstract}

Palavras-chave: desempenho agrícola; perfil do produtor; estratégia.

Abstract: Based on the Resource-Based View (VBR) theory, this work analyzed how the internal resources of the rural sugarcane properties in the states of Mato Grosso do Sul and Goiás influence their agricultural performance. It was an applied exploratory study, involving a field research with 148 rural producers. The quantitative analysis revealed differences between the sugarcane production patterns in the two study regions. Keywords: agricultural performance; producer profile; strategy.

Resumen: En base a la teoría de la Visión Basada en Recursos (VBR), este trabajo analizó cómo los recursos internos de las propiedades rurales de caña de azúcar en los estados de Mato Grosso do Sul y Goiás influencian su desempeño agrícola. Fue un estudio aplicado de carácter exploratorio, involucrando una investigación de campo con 148 productores rurales. El análisis cuantitativo reveló diferencias entre los patrones de producción de caña de azúcar en las dos regiones de estudio.

Palabras clave: rendimiento agrícola; perfil del productor; estrategia.

\section{INTRODUÇÃO}

O Brasil vivenciou, a partir do ano de 2003, uma expressiva expansão da produção de canade-açúcar (Saccharum officinarum), a qual foi guiada pelo aumento da demanda por etanol, em função do desenvolvimento do carro bicombustível (flex) e do interesse internacional em busca de fontes de energias alternativas (GILIO; MORAES, 2016).

Entre 2003 e 2016, a área plantada com cana-de-açúcar praticamente duplicou no país, incorporando cerca de novos 4,9 milhões de hectares (IBGE, s.d.). Tal processo alterou a representatividade das regiões que cultivam a cana-de-açúcar. Segundo Meurer, Shikida e Vian (2015) e Demattê et al. (2014), o Centro-Oeste é a região em que a produção de cana-de-açúcar mais se expande, assumindo o papel de segunda maior região produtora do país, ultrapassando a tradicional região Nordeste.

\footnotetext{
${ }^{1}$ Universidade Federal de São Carlos (UFSCar), São Carlos, São Paulo, Brasil.

${ }^{2}$ Universidade Estadual Paulista (UNESP), Tupã, São Paulo, Brasil.

${ }^{3}$ Kansas State University (KSU), Manhattan, Kansas, Estados Unidos.
} 
Destacam-se nessa expansão os estados de Mato Grosso do Sul (MS) e Goiás (GO), que obtiveram, no período entre 2003 e 2016, um crescimento da área plantada com cana-de-açúcar na ordem, respectivamente, de 446\% e 454\%, enquanto a taxa nacional esteve em 91\% (IBGE, s.d.).

De acordo com Strachman e Pupin (2011) e Castro et al. (2010), a expressiva expansão da cana-de-açúcar na última década para a região Centro-Oeste brasileira atendeu a uma demanda crescente de bioenergia, tanto do mercado nacional quanto do mercado internacional.

Granco et al. (2015) afirmam que a expansão do setor canavieiro nos estados de MS e GO é atribuída, principalmente, às políticas governamentais de apoio ao setor, ao aumento dos preços de terras nas regiões produtoras tradicionais e ao endurecimento das leis ambientais. Nesse contexto, são incluídas as boas condições edafoclimáticas nos estados de MS e GO; preços de terras inferiores do que os praticados no estado de São Paulo; propriedades com áreas mais extensas e planas, que facilitam as operações por meio da mecanização; e o fato de serem regiões localizadas próximas aos principais mercados consumidores, como o estado de São Paulo.

Considerando que o recente processo de expansão do cultivo da cana-de-açúcar em regiões de fronteira agrícola se deu por meio de um sistema produtivo moderno e tecnificado, advindo de uma trajetória efetiva de pesquisa no país, poder-se-ia supor que essas novas regiões apresentariam elevados índices de desempenho.

Entretanto a perspectiva promissora de crescimento da cultura da cana-de-açúcar na região Centro-Oeste tem enfrentado gargalos que precisam ser explorados e analisados. Embora os aumentos da área de produção e da quantidade produzida de cana-de-açúcar mereçam destaque, não se tem a mesma consideração quando analisada a sua produtividade agrícola recente (NYKO et al., 2013).

Apesar de a expansão da produção de cana-de-açúcar para a indústria sucroenergética ser resultante de decisões de nível macro (políticas econômicas e industriais), é fundamental que se considere o resultado de um conjunto de decisões de produtores sobre o melhor uso dos recursos disponíveis.

Nesse contexto, a análise dos aspectos que influenciam a produtividade agrícola envolve, além dos fatores climáticos, econômicos e políticos, elementos da firma, como o perfil do produtor rural, as características da propriedade, a tecnologia adotada, o sistema de produção e, principalmente, as decisões sobre o uso dos recursos envolvidos nas propriedades rurais.

Assim, torna-se relevante compreender quais são os fatores determinantes na composição dos indicadores de desempenho de produtividade da cana-de-açúcar das propriedades rurais dos estados de MS e GO, para subsidiar a proposição de ações em busca de maiores produtividades.

A corrente teórica denominada Visão Baseada em Recursos (VBR) permite a compreensão do desempenho das organizações em função da análise dos recursos internos. De acordo com Wernerfelt (1984), Barney (1991) e Peteraf (1993), as organizações são coleções diferentes de ativos tangíveis e intangíveis, e a chave para a formulação da estratégia está em compreender as relações entre recursos, capacidades, vantagem competitiva e lucratividade. Carvalho, Prevot e Machado (2014) afirmam que, embora disseminado para o estudo de empresas urbanas, o uso dessa teoria para a análise de propriedades rurais é adequado e promissor, avançando no conhecimento dos recursos internos.

Portanto o objetivo deste trabalho é analisar, a partir do arcabouço teórico da VBR, como os recursos internos das propriedades rurais de cana-de-açúcar nos estados de MS e GO influenciam seu desempenho agrícola. 


\section{VISÃO BASEADA EM RECURSOS (VBR) EM PROPRIEDADES RURAIS}

O uso da teoria da Visão Baseada em Recursos (VBR) possibilita ao agricultor diferentes percepções sobre as mudanças estruturais e sociais de uma propriedade rural. Permite compreender como o uso dos recursos internos da propriedade contribui para a definição da sua estratégia de crescimento. Os recursos em geral são formados por ativos tangíveis, como: equipamentos, maquinários, terras, entre outros; e intangíveis, como: conhecimento, cultura, aprendizado, entre outros. Esses recursos internos são controlados pelo gestor (agricultor), de forma a agregar valor aos seus produtos e serviços (WERNERFELT, 1984; BOEHLJE, 1999; KING et al., 2010).

Um dos pressupostos da VBR é que as empresas são heterogêneas quanto aos seus recursos e capacidades. Portanto essa teoria ajuda a explicar o uso dos vários tipos de recursos relacionados às empresas (fazendas), em seu processo de exploração de novas estratégias (DIERICKX; COOL, 1989; BARNEY, 1991; GRANDE, 2011). Explorar e desenvolver novas estratégicas significa desenvolver recursos em atendimento às novas necessidades da fazenda, satisfazendo, por sua vez, o mercado. Portanto desenvolver um recurso significa dar um novo olhar para o mesmo recurso ou mudá-lo fisicamente. Em resumo, o valor somente é criado quando os recursos são trabalhados, avaliados, desenvolvidos para o ambiente em que a fazenda está inserida (PRIEM; BUTLER, 2001; SIRMON; HITT; IRELAND, 2007).

Para Hennessy (2006) e Mann et al. (2014), o processo que envolve a tomada de decisão sobre o uso da terra pelos agricultores pode ser considerado como um problema de maximização, em que se busca o melhor rendimento desse fator de produção e, consequentemente, o maior retorno econômico possível (KELLERMAN, 1989; O'KELLY; BRYAN, 1996). Segundo os autores, existem diversos fatores que afetam o valor de locação do uso da terra agrícola, tais como: sua distância dos mercados compradores, o custo de transporte até a propriedade, o preço de mercado da terra e o custo local de se produzir. A partir da análise desses fatores, o agricultor deve decidir qual uso da terra pode maximizar seus lucros.

O emprego da VBR em propriedades rurais foi mapeado por Carvalho, Prevot e Machado (2014), que destacam a complexidade e multidimensionalidade da exploração agrícola. Os autores estabelecem, a partir dos estudos identificados, os recursos estratégicos internos.

[...] os recursos estratégicos para propriedades rurais são destacadamente os intangíveis, mais presentes nos recursos organizacionais e humanos [...]. Esses recursos têm a capacidade de prover condições de melhor usufruir de outros recursos (físicos, tecnológicos, financeiros e reputacionais), promovendo a sinergia e maiores ganhos nas organizações. (CARVALHO; PREVOT; MACHADO, 2014, p. 515).

\section{METODOLOGIA}

Os dados utilizados para a análise deste trabalho estão vinculados a um banco de dados de um projeto interinstitucional ${ }^{4}$. A coleta dos dados ocorreu durante o mês de julho de 2014. Os dados levantados são oriundos de questões sobre o uso dos recursos tangíveis e intangíveis, referentes ao processo de produção da cana-de-açúcar, bem como questões qualitativas, que

\footnotetext{
${ }^{4}$ Projeto "Direct and indirect drivers of land cover change in the Brazilian Cerrado: the role of public policy, market forces, and sugarcane expansion", financiado pela National Science Foundation (NSF).
} 
compreendem o processo de tomada de decisão pelos produtores rurais dos estados de MS e GO.

O processo de indicação e seleção dos produtores ocorreu a partir de apoiadores das organizações de classe dos diferentes municípios do estado de MS e GO, tais como: associações de plantadores de cana e sindicados rurais. Aos agricultores que concordaram em participar da pesquisa, foi aplicado um formulário abordando informações como: perfil do produtor rural (escolaridade); dados da propriedade (tamanho da propriedade); dados de produção (produtividade, adoção de irrigação, adoção de colheita mecanizada, adoção de variedade de cana adaptada ao Cerrado); tipo de contrato com a usina (arrendamento, parceria e fornecimento); e uso anterior da terra (substituição de outras culturas ou de pastagem por cana).

A definição dos locais (municípios) para a coleta de dados teve como base dois critérios: a) localização geográfica da produção de cana-de-açúcar em 2012, de acordo com o Projeto CANASAT, do Instituto Nacional de Pesquisa Espacial (INPE); b) municípios que se destacaram na evolução da produção de cana-de-açúcar, segundo estimativas da Produção Agrícola Municipal (PAM) de 2013 (IBGE, s.d.).

No total, foram realizadas 148 entrevistas, sendo 83 aplicadas aos produtores do estado de $\mathrm{GO}$ e outras 65 aos produtores do estado de MS. Em Mato Grosso do Sul, as pesquisas foram realizadas em 13 municípios; já no estado de Goiás foram aplicadas em 17. Tais municípios representavam, em 2013, um total de aproximadamente 4\% e 5\% da área total de plantações de cana-de-açúcar em GO e MS, respectivamente. A distribuição dos respondentes por localidade está apresentada na Tabela 1.

Tabela 1 - Número de produtores entrevistados, por município, em MS e GO

\begin{tabular}{lclc}
\hline \multicolumn{2}{c}{ MATO GROSSO DO SUL } & \multicolumn{2}{c}{ GOIÁS } \\
\hline \multicolumn{1}{c}{ Município } & N. de Respondentes & \multicolumn{1}{c}{ Município } & N. de Respondentes \\
\hline Angélica & 13 & Bom Jesus de Goiás & 2 \\
Brasilândia & 2 & Cachoeira Dourada & 9 \\
Caarapó & 1 & Caçu & 1 \\
Campo Grande & 1 & Edéia & 18 \\
Costa Rica & 11 & Goiatuba & 10 \\
Deodápolis & 4 & Gouvelândia & 2 \\
Dourados & 1 & Inaciolândia & 6 \\
Ivinhema & 6 & Indiara & 1 \\
Juti & 1 & Itumbiara & 5 \\
Maracaju & 7 & Jataí & 2 \\
Nova Alvorada do Sul & 5 & Joviânia & 1 \\
Rio Brilhante & 12 & Montividiu & 2 \\
São Gabriel do Oeste & 1 & Morrinhos & 3 \\
& & Paraúna & 2 \\
\multicolumn{1}{c}{ Total } & Quirinópolis & 14 \\
& & Rio Verde & 3 \\
& & Vicentinópolis & Total \\
\hline
\end{tabular}

Fonte: Elaborada pelos autores. 
A escolha das variáveis (Quadro 1) partiu da suposição, com base nas discussões teóricas e empíricas evidenciadas, de que parâmetros que abordam decisões do uso de recursos e capacidades de gestão sobre as propriedades rurais e seus proprietários afetam a produtividade de cana-de-açúcar dos estados de MS e GO.

Caracterizado como um estudo estatístico não experimental, ou observacional, esse trabalhou adotou como métodos de análise a estatística descritiva dos dados e a tabulação cruzada. A ferramenta utilizada para a análise dos dados foi o software SPSS 22.0.

Quadro 1 - Variáveis selecionadas para a pesquisa

\begin{tabular}{|llcc|}
\hline & Variável (Recursos Internos)* & Medida & Recurso** \\
\hline V1 & Produtividade da Cana-de-Açúcar & ton/ha ou kg ATR/ton & Tecnológico \\
V2 & Escolaridade do Produtor Rural & Faixas de estudo & Humano \\
V3 & Tamanho da Propriedade Rural & Hectares & Físico \\
V4 & Mecanização na Produção & Sim/não & Tecnológico \\
V5 & Uso da Terra anterior à Cana & Pastagem/Lavoura & Físico \\
V6 & Variedade de Cana para o Cerrado & Sim/não & Tecnológico \\
V7 & Irrigação na Produção de Cana & Sim/não & Tecnológico \\
V8 & Contrato entre Produtor e Usina & Arrendamento/Parceria/Fornecimento & Organizacional \\
V9 & Participação em Org. de classe & Sim/não & Reputacional \\
\hline
\end{tabular}

* Variáveis obtidas no banco de dados do projeto mãe.

** Classificação a partir de Carvalho, Prevot e Machado (2014).

Fonte: Elaborado pelos autores.

A estatística descritiva dos dados se baseou em parâmetros selecionados a partir do banco de dados, sendo esses relacionados direta ou indiretamente à produtividade agrícola da canade-açúcar. O uso da tabulação cruzada comparou estatisticamente (Teste do Qui-Quadrado) a frequência de respostas das variáveis supracitadas para os dois grupos de respondentes, ou seja, dos entrevistados dos estados de MS e GO.

A tabulação cruzada é a distribuição de frequência de respostas de dois ou mais conjuntos de variáveis. Isso significa que tabulamos as respostas para cada grupo e as comparamos. A análise do qui-quadrado permite-nos testar se há diferenças estatísticas entre grupos.

$[\ldots]$

A estatística qui-quadrado é utilizada para testar a significância estatística entre as distribuições de frequência de dois ou mais grupos. [...] A hipótese nula é de que não há diferença. (HAIR et al., 2005, p. 292-3).

Segundo Lay e Reis (2005), no teste de tabulação cruzada existe uma relação entre as variáveis quando os valores de $x^{2}$ são estatisticamente significativos (valores iguais ou menores do que 0,05$)$. Contudo, ressaltam os autores, a relação estatística pode ser apenas uma coincidência, devendo esta ser confrontada com a possibilidade da veracidade desta relação, tendo como base o conhecimento sobre a lógica de relação entre elas. Dessa forma, não se faz prudente apenas descartar dados em função da inexistência de relações estatisticamente significativas. 


\section{RESULTADOS}

Para cada uma das variáveis (recursos internos) selecionadas para o desenvolvimento desta pesquisa, foi descrita a frequência de respostas para os dois grupos de respondentes (MS e GO), bem como verificada a existência de diferença significativa entre eles.

\section{a) Produtividade da cana-de-açúcar}

A avaliação da produtividade da cana-de-açúcar se dá por meio do cálculo em toneladas por hectare (ton/ha) ou em quilograma de ATR por tonelada de cana-de-açúcar (kg ATR/ton). A Tabela 2 identifica os dados médios das propriedades entrevistadas nos estados de Mato Grosso do Sul e Goiás, bem como evidencia a análise comparativa por meio do Teste Qui-Quadrado (Tabulação Cruzada).

Tabela 2 - Comparação da produtividade média agrícola (ton/ha e kg ATR/ton) da cana-de-açúcar entre as propriedades pesquisadas em MS e GO e o respectivo Teste Qui-Quadrado

\begin{tabular}{ccccc}
\hline V1 & Produtividade & MS & Go & $\begin{array}{c}\text { Teste } \\
\text { Qui-Quadrado }\end{array}$ \\
\hline ton/ha & 94,3 & 100,8 & 0,815 \\
kg ATR/ton & 124,4 & 139,0 & $\mathbf{0 , 0 0 0}$ \\
\hline
\end{tabular}

Fonte: Elaborada pelos autores a partir dos dados da pesquisa.

Semelhante aos dados oficiais ${ }^{5}$, verifica-se, nesta pesquisa, um desempenho relativo maior do estado de GO em relação a MS, tanto em termos de ton/ha quanto em kg ATR/ton. Entretanto a análise estatística dos dados revelou diferença significativa somente em termos de kg ATR/ tonelada, evidenciando uma maior produtividade do estado de GO em relação a MS.

\section{b) Escolaridade do produtor rural}

Foi avaliado o nível de escolaridade dos produtores rurais. A Tabela 3 categoriza os diferentes níveis de escolaridade dos produtores entrevistados, nos estados de MS e GO, bem como realiza a comparação estatística dessa variável.

Verificou-se que não há diferença significativa entre os grupos analisados. Assim, considerase como semelhante o grau de escolaridade dos produtores entrevistados entre os dois estados pesquisados. Nota-se a predominância do nível de escolaridade dos entrevistados com, no mínimo, o Ensino Médio completo.

\footnotetext{
${ }^{5}$ Dados institucionais (público e privado). De acordo com o IBGE (2017), as produtividades em ton/ha em MS e GO eram, em 2012, respectivamente, de 67,6 e 79,6. De acordo com o Centro Tecnológico Canavieiro (CTC, 2015), a produtividade em kg ATR/ton em MS e GO era, em 2012, respectivamente, de 125,8 e 134,8.
} 
Tabela 3 - Frequência relativa do nível de escolaridade dos produtores rurais entrevistados em MS e GO e o respectivo Teste Qui-Quadrado

\begin{tabular}{llcccc}
\hline V2 & Nível de Escolaridade & MS & Go & $\begin{array}{c}\text { Teste } \\
\text { Qui-Quadrado }\end{array}$ & $\mathbf{0 , 2 5 8}$ \\
\hline Até a quarta série & $14 \%$ & $19 \%$ & \\
Até a oitava série & $5 \%$ & $9 \%$ & \\
Ensino Médio completo & $43 \%$ & $34 \%$ & \\
Ensino Superior & $27 \%$ & $29 \%$ & \\
Pós-graduação & $11 \%$ & $9 \%$ & \\
\hline
\end{tabular}

Fonte: Elaborada pelos autores a partir dos dados da pesquisa.

Tal situação se destaca diante da média do país. De acordo com o IBGE (2006), a escolaridade do produtor rural brasileiro apresenta a seguinte configuração: 39,1\% são analfabetos ou sabem ler e escrever, mas não têm nenhum grau de escolaridade; 42,4\% têm Ensino Fundamental incompleto; 7,3\% têm Ensino Técnico Profissionalizante ou Ensino Médio; e apenas 2,8 \% têm nível superior completo.

É importante destacar que os dados oficiais englobam produtores rurais de modo geral e que os dados da pesquisa de campo abordam especificamente os produtores de cana-de-açúcar, o que sem dúvida segmenta um perfil diferente.

\section{c) Tamanho da propriedade rural}

De acordo com os dados obtidos na pesquisa de campo, o tamanho médio das propriedades com cana-de-açúcar entrevistadas é de 883 hectares, sendo que as propriedades de MS apresentaram, em média, um pouco mais que o dobro do tamanho das propriedades de GO. A Tabela 4 apresenta os dados pesquisados, bem como a análise estatística comparativa.

Tabela 4 - Tamanho médio (ha) das propriedades com cana-de-açúcar entrevistadas em MS e GO e o respectivo Teste Qui-Quadrado

\begin{tabular}{cccccc}
\hline V3 & $\begin{array}{c}\text { Tamanho Médio das } \\
\text { Propriedades }\end{array}$ & MS & GO & $\begin{array}{c}\text { Teste } \\
\text { Qui-Quadrado }\end{array}$ & 0,006 \\
\cline { 1 - 3 } & Hectares & 1.219 & 568 & & \\
\hline
\end{tabular}

Fonte: Elaborada pelos autores a partir dos dados da pesquisa de campo.

A análise estatística comprova a diferença significativa entre o tamanho das propriedades entrevistadas em MS e GO; ou seja, em MS, as propriedades são maiores do que em GO. Considera-se que essa variável contribui para explicar o uso intensivo de mecanização no processo de plantio e/ou colheita da cultura da cana-de-açúcar. Quanto maior a área agricultável, maior a propensão do uso da mecanização agrícola. 


\section{d) Adoção de mecanização na produção}

Ao considerar o nível de mecanização da colheita de cana-de-açúcar nas propriedades rurais pesquisadas, verifica-se uma alta adoção tecnológica em ambos os estados. A Tabela 5 sistematiza os dados obtidos a partir das entrevistas e realiza a análise estatística.

Tabela 5 - Frequência do uso da mecanização da colheita de cana-de-açúcar das propriedades rurais pesquisadas em MS e GO e o respectivo Teste Qui-Quadrado

\begin{tabular}{cccccc}
\hline V4 & Mecanização da colheita & MS & GO & $\begin{array}{c}\text { Teste } \\
\text { Qui-Quadrado }\end{array}$ & $\mathbf{0 , 7 8 3}$ \\
\hline Nível de adoção & $94,3 \%$ & $82,7 \%$ & \\
\hline
\end{tabular}

Fonte: Elaborada pelos autores a partir dos dados da pesquisa de campo.

Não há diferença significativa na adoção da mecanização entre os estados pesquisados. Entretanto, de acordo com Nyko et al. (2013) e Demattê (2012), embora contraintuitivo, o elevado uso da mecanização nas áreas pesquisadas pode trazer efeitos negativos à produtividade agrícola da cana-de-açúcar, por meio do aumento de tráfego na lavoura que acarreta, entre outros danos, o pisoteamento e, consequentemente, a compactação do solo.

\section{e) Uso da terra anterior ao cultivo da cana-de-açúcar}

Considerou-se importante identificar para cada um dos estados analisados qual era a atividade agropecuária predominantemente utilizada em período anterior à implantação da cultura da cana-de-açúcar. A Tabela 6 mostra a frequência do uso da terra anterior ao cultivo de cana-de-açúcar com pastagem ou lavoura nos estados de MS e GO e o respectivo Teste Qui-Quadrado.

Por meio das frequências relativas, percebe-se um padrão diferente entre os estados de MS e GO. Enquanto no estado de MS a maioria das propriedades com cana-de-açúcar tinha como atividade anterior a pecuária, no estado de GO a maioria das propriedades que atualmente cultivam cana-de-açúcar ocupa áreas anteriormente destinadas às lavouras agrícolas, como soja, milho e algodão.

Tabela 6 - Frequência relativa do uso da terra anterior ao cultivo de cana-de-açúcar com pastagem ou lavoura nos estados de MS e GO e o respectivo Teste Qui-Quadrado

\begin{tabular}{ccccc}
\hline V5 & $\begin{array}{c}\text { Uso da terra anterior ao cultivo da } \\
\text { cana-de-açúcar }\end{array}$ & MS & GO & $\begin{array}{c}\text { Teste } \\
\text { Qui-Quadrado }\end{array}$ \\
\hline Pastagem & $69 \%$ & $37 \%$ & $\mathbf{0 , 0 0 6}$ \\
Lavoura & $31 \%$ & $63 \%$ & 0,172 \\
\hline
\end{tabular}

Fonte: Elaborada pelos autores a partir dos dados da pesquisa de campo.

A análise estatística, entretanto, revelou diferença significativa entre os estados pesquisados apenas quando o uso anterior da terra era com pastagem. Portanto afirma-se estatisticamente que, diferentemente de GO, nas propriedades entrevistadas de MS a cana substituiu áreas anteriormente cobertas por pastagens, utilizadas para a atividade pecuária. 


\section{f) Variedade de cana para o Cerrado}

Ao analisar a possível adoção de variedades de cana-de-açúcar desenvolvidas especificamente para a região do Cerrado, percebeu-se uma baixa adesão desse componente tecnológico de produção por parte dos produtores entrevistados de ambos os estados, conforme se observa na Tabela 7.

Tabela 7 - Frequência na adoção de variedade de cana-de-açúcar desenvolvida para o Cerrado nas propriedades entrevistadas de MS e GO e o respectivo Teste Qui-Quadrado

\begin{tabular}{cccccc}
\hline V6 & $\begin{array}{c}\text { Possui variedade de cana } \\
\text { para Cerrado }\end{array}$ & MS & GO & $\begin{array}{c}\text { Teste } \\
\text { Qui-Quadrado }\end{array}$ & 0,809 \\
\hline $\operatorname{Sim}$ & $12 \%$ & $14 \%$ & & \\
\hline
\end{tabular}

Fonte: Elaborada pelos autores a partir dos dados da pesquisa de campo.

Não foi identificada diferença significativa entre o padrão de adoção de variedades adaptadas ao Cerrado entre os produtores entrevistados de MS e GO. Para aqueles produtores que afirmaram não adotar, as anotações de campo revelam que as variedades comumente empregadas são as desenvolvidas para o estado de São Paulo, tradicional produtora de canade-açúcar, especialmente a RB 867515 e a SP 81-3250.

Argumenta-se que o uso intensivo de variedades de cana específicas para o Cerrado contribuiria para o aumento dos índices de produtividade da cana nas respectivas regiões de MS e GO.

\section{g) Uso de irrigação na produção de cana}

Os dados obtidos na pesquisa revelam que é baixa a adoção do uso de sistema de irrigação na atividade de produção agrícola da cana-de-açúcar. Apenas 7\% dos respondentes afirmaram utilizar algum sistema de irrigação (Tabela 8).

Tabela 8 - Frequência do uso de irrigação no cultivo de cana-de-açúcar pelos produtores entrevistados em MS e GO e o respectivo Teste Qui-Quadrado

\begin{tabular}{cccccc}
\hline V7 & $\begin{array}{c}\text { Usa irrigação na lavoura de } \\
\text { cana }\end{array}$ & MS & GO & $\begin{array}{c}\text { Teste } \\
\text { Qui-Quadrado }\end{array}$ & $\mathbf{0 , 2 6 1}$ \\
\hline Sim & $6 \%$ & $8 \%$ & & \\
\hline
\end{tabular}

Fonte: Elaborada pelos autores a partir dos dados da pesquisa de campo.

Além da constatação da baixa adoção, não foi encontrada diferença significativa entre os produtores entrevistados de MS e GO. Evidências empíricas mostraram que as propriedades que adotavam sistema de irrigação (fertirrigação) foram aquelas localizadas próximas às usinas. Nesses casos, geralmente, a vinhaça (subproduto das usinas) é encaminhada por tubulações (pela gravidade) até as áreas de produção de cana, contribuindo como adubo para o solo. 


\section{h) Tipos de contrato entre produtor e usina}

Os tipos de contratos entre os produtores rurais e as usinas, verificados durante a pesquisa de campo, tiveram as seguintes definições:

i) Arrendamento de terra: contrato por meio do qual o proprietário concede à usina local o uso de sua propriedade rural para o cultivo da cana por um valor fixo pago periodicamente;

ii) Parceria agrícola: contrato no qual o proprietário concede o uso de sua propriedade para o cultivo da cana e em troca recebe uma percentagem da produção produzida naquela terra;

iii) Contrato de fornecimento: contrato no qual o fazendeiro concorda em fornecer cana-de-açúcar à usina.

A Tabela 9 apresenta a frequência do uso de diferentes tipos de contratos entre os produtores rurais e as usinas, identificados pela pesquisa de campo, e a respectiva análise comparativa.

Tabela 9 - Frequência do uso de contratos (arrendamento, parceria e fornecimento) entre os produtores entrevistados e as usinas de cana-de-açúcar em MS e GO e o respectivo Teste QuiQuadrado

\begin{tabular}{cccccc}
\hline V8 & Tipos de Contrato & MS & Go & $\begin{array}{c}\text { Teste } \\
\text { Qui-Quadrado }\end{array}$ & $\mathbf{0 , 8 7 6}$ \\
\hline & Arrendamento & $11 \%$ & $23 \%$ & & \\
& Parceria & $\mathbf{5 8 \%}$ & $4 \%$ & & \\
Fornecimento & $31 \%$ & $\mathbf{7 3 \%}$ & & \\
\hline
\end{tabular}

Fonte: Elaborada pelos autores a partir dos dados da pesquisa de campo.

As frequências revelam que, no estado de GO, há predominância (73\%) de contratos de fornecimento, enquanto em MS a concentração está nos contratos de parceria (58\%). Entretanto não se puderam comprovar estatisticamente diferenças significativas entre o uso dos diferentes tipos de contrato em MS e GO.

Porém observou-se na pesquisa de campo que os produtores que afirmaram ter um contrato de parceria, na realidade, adotavam um mecanismo de arrendamento. Tal fato decorre das estratégias praticadas pelas usinas que formalmente aplicam o mecanismo de parceria, enquanto, na prática, a relação se caracteriza como arrendamento, em função principalmente de ganhos tributários do mecanismo formal.

\section{i) Participação em organizações de classe}

Ao se analisar a participação dos produtores rurais de ambos os estados em organizações de classe (Tabela 10), nota-se uma adesão superior a 85\%. Esses produtores afirmam participar de sindicatos, associações, cooperativas, entre outros. Não foi verificada diferença significativa no perfil de participação em organizações de classe dos produtores entrevistados de MS e GO. 
Tabela 10 - Frequência de participação em organizações de classe dos produtores entrevistados de MS e GO e o respectivo Teste Qui-Quadrado

\begin{tabular}{cccccc}
\hline V9 & $\begin{array}{c}\text { Participação em } \\
\text { organizações de classe }\end{array}$ & MS & GO & $\begin{array}{c}\text { Teste } \\
\text { Qui-Quadrado }\end{array}$ & $\mathbf{0 , 2 7 3}$ \\
\hline $\operatorname{Sim}$ & $85 \%$ & $88 \%$ & & \\
\hline
\end{tabular}

Fonte: Elaborada pelos autores a partir dos dados da pesquisa.

Considera-se que a participação em organizações de classe tende a contribuir para a melhoria do desempenho agrícola, por meio de compartilhamento de informações e ganhos econômicos e sociais da ação coletiva.

\section{j) Sistematização comparativa}

A partir da análise estatística dos dados, os recursos internos das propriedades de cana-de-açúcar analisadas para os estados de MS e GO foram sistematizados e comparados (Quadro 2).

Quadro 2- Análise comparativa dos recursos internos entre os entrevistados de MS e GO

\begin{tabular}{|c|c|c|c|c|}
\hline & $\begin{array}{c}\text { Variável } \\
\text { (Recursos Internos) }\end{array}$ & MS & Recurso & $\begin{array}{c}\text { Diferença } \\
\text { Significativa* }\end{array}$ \\
\hline V1 & $\begin{array}{l}\text { Produtividade da cana-de- } \\
\text { açúcar }\end{array}$ & Menor & Tecnológico & $x$ \\
\hline V2 & Escolaridade do produtor & Nivel semelhante & Humano & \\
\hline V3 & Tamanho da propriedade & Menor & Físico & $x$ \\
\hline V4 & Mecanização da produção & Alta adoção & Tecnológico & \\
\hline V5 & $\begin{array}{l}\text { Uso da terra anterior à cana } \\
\text { (pastagem) }\end{array}$ & Não & Físico & $x$ \\
\hline V6 & $\begin{array}{l}\text { Variedade de cana para o } \\
\text { Cerrado }\end{array}$ & Baixa adoção & Tecnológico & \\
\hline V7 & $\begin{array}{l}\text { Irrigação na produção de } \\
\text { cana }\end{array}$ & Baixa adoção & Tecnológico & \\
\hline V8 & $\begin{array}{l}\text { Contrato entre produtor e } \\
\text { usina }\end{array}$ & Arrendamento Fornecimento & Organizacional & \\
\hline V9 & $\begin{array}{l}\text { Participação em org. de } \\
\text { classe }\end{array}$ & Alta participação & Reputacional & \\
\hline
\end{tabular}

* Teste do Qui-Quadrado

Fonte: Elaborado pelos autores.

Os resultados demonstraram que os recursos internos referentes à escolaridade, à mecanização, ao uso de variedade adaptada, ao uso de irrigação, ao tipo de contrato e à participação em organizações de classe não apresentaram diferenças significativas entre os estados de MS e GO. Entretanto ressalta-se que, a partir de evidências empíricas, os autores deste trabalho consideram um padrão diferente entre MS e GO, no que se refere ao tipo de contrato utilizado entre o produtor e a usina. Para esses pesquisadores, o contrato predominante em MS é o arrendamento e, em GO, é o fornecimento. 
Nesse contexto, pode-se afirmar estatisticamente que as propriedades de MS apresentaram produtividade (kg ART/ton) menor do que GO em propriedades com extensõès de terra maiores, as quais eram usadas, predominantemente, para pastagens e, consequentemente, para a atividade pecuária. Acrescenta-se a esse padrão a predominância do uso do contrato de arrendamento (evidências empíricas).

De maneira oposta a esse padrão, afirma-se que as propriedades de GO apresentam uma produtividade maior e em extensões de área menores do que em MS. Sugere-se ainda que, nesse estado, o cultivo de cana-de-açúcar substituiu áreas que anteriormente eram dedicadas às lavouras e que o contrato predominante é o fornecimento.

\section{DISCUSSÃO DOS RESULTADOS}

A partir da análise dos dados e da observação empírica na pesquisa de campo, discutemse os diferentes padrões de cultivo de cana-de-açúcar em MS e GO, revelando os fatores que promovem seus desempenhos.

Em MS, a expansão da atividade agrícola canavieira ocorreu, predominantemente, em áreas que anteriormente eram destinadas à atividade pecuária. A decisão do uso dos recursos por parte dos proprietários rurais se deu ao comparar a lucratividade do uso da terra explorada na pecuária extensiva e as propostas das usinas, principalmente por contratos de arrendamento. Considerando que a maior parte das áreas de pastagens substituídas estava degradada ou com reduzido padrão de manejo, mesmo com as correções efetuadas, o solo não estava em condições para a implantação da cultura da cana com alto desempenho.

Os proprietários rurais e tomadores de decisão apresentam perfil de pecuaristas tradicionais, com pouco ou nenhum conhecimento ou experiência em atividades agrícolas; portanto com perfil investidor e de pouca atuação no dia a dia da atividade do campo. Muitos deles não residem na propriedade, no município e/ou no estado. Com a oportunidade de mudança para a atividade canavieira, opta-se, preferencialmente, pelo contrato de arrendamento, que não requer envolvimento na atividade e possibilita retornos certos e predefinidos.

Em MS, o tamanho médio das propriedades é maior do que no estado vizinho, o que, aliado às condições de baixa declividade, possibilita um alto nível de mecanização. Além da mecanização ser realizada por terceiros (arrendamento), sem o monitoramento direto do proprietário, há os efeitos negativos dessa tecnologia para com a produtividade dessa cultura.

Quando se analisa o padrão de produção de cana-de-açúcar no estado de GO, verifica-se um nível de produtividade maior do que encontrado no estado de MS. Em geral, sugere-se que a implantação da cultura da cana-de-açúcar se deu, predominantemente, em áreas anteriormente destinadas à produção de lavouras temporárias, como soja, milho e algodão. No que se refere às condições do solo, supõe-se que tais áreas já apresentavam condições adequadas para o uso intensivo e de alto desempenho da atividade agrícola.

Os proprietários e tomadores de decisão dessas terras eram produtores de commodities, com maior envolvimento na atividade agrícola, com conhecimentos técnicos e apurados e com dedicação no dia a dia do campo. Com esse perfil, opta-se pelo contrato de fornecimento, que, apesar de apresentar riscos, oferece maiores oportunidades de ganhos advindos da conquista de níveis superiores de produtividade. 
Embora as propriedades apresentem um tamanho médio menor do que em MS, as condições de baixa declividade também permitem um alto nível de mecanização. Entretanto, nesse caso, o engajamento do produtor com a atividade agrícola, a busca pelo maior desempenho em função do tipo de contrato (fornecimento) e o monitoramento do uso da tecnologia reduzem, de certa forma, os efeitos negativos da mecanização.

\section{CONCLUSÕES FINAIS}

Este trabalho analisou, a partir do arcabouço teórico da VBR, como os recursos internos das propriedades rurais de cana-de-açúcar nos estados de MS e GO influenciam seu desempenho agrícola.

As abordagens teóricas e empíricas proporcionaram meios de explorar variáveis importantes na análise da produtividade. Variáveis essas relacionadas com o uso dos recursos tangíveis e intangíveis das propriedades rurais, bem como a capacidade de gestão e adoção de tecnologias agrícolas.

Identificou-se diferença significativa entre a produtividade de MS e GO, respaldada estatisticamente pelos recursos: tamanho da propriedade e uso anterior da terra. Além desses, o tipo de contrato entre o produtor e a usina também é evidenciado pelos autores. Nesse contexto, conclui-se que os recursos físicos e organizacionais são destacadamente estratégicos para o desempenho das propriedades analisadas.

Por fim, considera-se que este trabalho contribui para a interlocução entre as teorias estratégicas, largamente utilizadas para estudo de organizações urbanas, e a pesquisa de desempenho de empreendimentos rurais.

\section{REFERÊNCIAS}

BARNEY, J. Firm resources and sustained competitive advantage. Journal of Management, v. 17, n. 1, p. 99-120, 1991.

BOEHLJE, M. Structural changes in the agricultural industries: how do we measure, analyze and understand them? American Journal of Agricultural Economics, v. 81, n. 5, p. 1028-41, 1999.

CARVALHO, D. M.; PREVOT, F.; MACHADO, J. A. D. O uso da teoria da visão baseada em recursos em propriedades rurais: uma revisão sistemática da literatura. Revista de Administração, São Paulo, v. 49, n. 3, p. 506-18, jul./set. 2014. Disponível em: http://www.scielo.br/scielo.php?script=sci_arttext\&pid $=$ S0080-21072014000300006. Acesso em: 13 fev. 2020.

CASTRO, S. S.; ABDALA, K.; SILVA, A. A.; BÔRGES, V. M. S. A expansão da cana-de-açúcar no Cerrado e no Estado de Goiás: elementos para uma análise espacial do processo. Boletim Goiano de Geografia, Goiânia, v. 30, n. 1, p. 171-91, jan./jun. 2010. Disponível em: https://repositorio.bc.ufg.br/xmlui/handle/ ri/1444?show=full. Acesso em: 13 fev. 2020.

DEMATTÊ, J. L. I. Variedades de cana estão devendo. Idea News-Cana \& Indústria, Ribeirão Preto, SP, v. 11, n. 141, p. 16-24, 2012.

DEMATTÊ, J. A. M.; DEMATTÊ, J. L. I.; ALVES, E. R.; BARBOSA, R. N.; MORELLI, J. L. Precision agriculture for sugarcane management: a strategy applied for brazilian conditions. Acta Scientiarum Agronomy, Maringá, PR, v. 36, n. 1, p. 111-7, jan./mar. 2014. DOI: http://dx.doi.org/10.4025/actasciagron.v36i1.17664 
DIERICKX, I.; COOL, K. Asset stock accumulation and sustainability of competitive advantage. Management Science, v. 35, n. 12, p. 1504-11, 1989.

GILIO, L.; MORAES, M. A. F. D. Sugarcane industry's socioeconomic impact in São Paulo, Brazil: a spatial dynamic panel approach. Energy Economics, v. 58, p. 27-37, ago. 2016. DOI: https://doi.org/10.1016/j. eneco.2016.06.005

GRANCO, G.; CALDAS, M. M.; BERGTOLD, J. S.; SANT'ANNA, A. C. Exploring the policy and social factors fueling the expansion and shift of sugarcane production in the Brazilian Cerrado. GeoJournal, v. 82, n. 1, p. 63-80, ago. 2015. DOI: http://dx.doi.org/10.1007/s10708-015-9666-y

GRANDE, J. New venture creation in the farm sector: critical resources and capabilities. Journal of Rural Studies, v. 27, n. 2, p. 220-33, abr. 2011. DOI: https://doi.org/10.1016/j.jrurstud.2011.02.003

HAIR JR., J. F.; BABIN, B.; MONEY, A. H.; SAMOEUL, P. Fundamentos de métodos de pesquisa em administração. Porto Alegre: Bookman, 2005.

HENNESSY, D. A. On monoculture and the structure of crop rotations. American Journal of Agricultural Economics, v. 88, n. 4, p. 900-14, nov. 2006. DOI: http://www.jstor.org/stable/4123535

IBGE. Censo Agropecuário 2006. Disponível em: http://www.sidra.ibge.gov.br/bda/pecua/default. asp?z=t\&o=24\&i=P. Acesso em: jul. 2016.

IBGE. Tabela 1612 - Área plantada, área colhida, quantidade produzida, rendimento médio e valor da produção das lavouras temporárias. [s.d.]. Disponível em: http://www.sidra.ibge.gov.br/bda/tabela/listabl. asp?c=1612\&z=p\&o=24. Acesso em: nov. 2017.

KELLERMAN, A. Agricultural location theory 1: basic models. Environment and Planning A: Economy and Space, v. 21, n. 10, p. 1381-96, out. 1989. DOI: https://doi.org/10.1068/a211381

KING, R. P.; BOEHLJE, M.; COOK, M. L.; SONKA, S. T. Agribusiness economics and management. American Journal of Agricultural Economics, v. 92, n. 2, p. 554-70, abr. 2010. DOI: https://doi.org/10.1093/ajae/ aaq009

LAY, M. C. D.; REIS, A. T. D. L. Análise quantitativa na área de estudos ambiente-comportamento. Ambiente construído: Revista da Associação Nacional de Tecnologia do Ambiente Construído, Porto Alegre, v. 5, n. 2, p. 21-36, abr./jun. 2005.

MANN, M. L.; KAUFMANN, R. K.; BAUER, D. M.; GOPAL, S.; NOMACK. M.; WOMACK, J. Y.; SOARES-FILHO, B. S. Pasture conversion and competitive cattle rents in the Amazon. Ecological economics, v. 97, p. 18290, jan. 2014. DOI: https://doi.org/10.1016/j.ecolecon.2013.11.014

MEURER, A. P. S.; SHIKIDA, P. F. A.; VIAN, C. E. F. Análise da agroindústria canavieira nos estados do Centro-Oeste do Brasil a partir da Matriz de Capacidades Tecnológicas. Revista de Economia e Sociologia Rural, Brasília, v. 53, n. 1, p. 159-78, jan./mar. 2015. DOI: http://dx.doi.org/10.1590/1234-567818069479005301009

NYKO, D.; VALENTE, M. S.; MILANEZ, A. Y.; TANAKA, A. K. R.; RODRIGUES, A. V. P. A evolução das tecnologias agrícolas do setor sucroenergético: estagnação passageira ou crise estrutural? BNDES Setorial, n. 37, p. 399-442, 2013. Disponível em: https://web.bndes.gov.br/bib/jspui/bitstream/1408/1503/2/A\%20 mar37_10_A\%20evolu\%c3\%a7\%c3\%a30\%20das\%20tecnologias\%20agr\%c3\%adcolas\%20do\%20setor_P. pdf. Acesso em: 13 fev. 2020. 
O'KELLY, M.; BRYAN, D. Agricultural location theory: von Thunen's contribution to economic geography. Progress in Human Geography, v. 20, n. 4, p. 457-75, 1996. DOI: https://doi. org/10.1177/030913259602000402

PETERAF, M. A. The cornerstones of competitive advantage: a resource based view. Strategic Management Journal, v. 14, n. 3, p. 179-91, mar. 1993.

PRIEM, R. L.; BUTLER, J. E. Tautology in the resource-based view and the implications of externally determined resource value: further comments. Academy of Management Review, v. 26, n. 1, p. 57-66, jan. 2001. DOI: http://www.jstor.org/stable/259394

SIRMON, D. G.; HITT, M. A., IRELAND, R. D. Managing firm resources in dynamic environments to create value: looking inside the black box. Academy of Management Review, v. 32, n. 1, p. 273-92, jan. 2007. DOI: http://www.jstor.org/stable/20159292

STRACHMAN, E.; PUPIN, G. M. The Brazilian sugar and alcohol sector: evolution, production chain and innovations. Cepal Review, Santiago, Chile, n. 103, p. 179-198, 2011.

WERNERFELT, B. A resource-based view of the firm. Strategic Management Journal, v. 5, n. 2, p. 171-80, abr./jun. 1984. DOI: https://doi.org/10.1002/smj.4250050207

\section{Sobre os autores:}

Roberto Bernardo: Doutor em Engenharia de Produção pela Universidade Federal de São Carlos (UFSCar). Mestre em Agronegócio e Desenvolvimento pela Universidade Estadual Paulista (UNESP). Engenheiro Mecânico graduado pela UNESP de Ilha Solteira. E-mail: betobernardo@ uol.com.br, Orcid: https://orcid.org/0000-0002-3140-9138

Wagner Luiz Lourenzani: Estágio Pós-Doutoral em Geografia na Kansas State University. Doutor em Engenharia de Produção pela Universidade Federal de São Carlos (UFSCar). Mestre em Ciência e Tecnologia de Alimentos pela Universidade Federal de Viçosa (UFV). Graduado em Engenharia de Alimentos pela UFV. Livre-Docente em Análise de Sistemas Agroindustriais pela Universidade Estadual Paulista (UNESP). Professor associado da Faculdade de Ciências e Engenharia da UNESP. Docente permanente do Programa de Pós-Graduação em Agronegócio e Desenvolvimento (PGAD). Pesquisador do Centro de Pesquisa em Administração e Agronegócio (CEPEAGRO/UNESP). E-mail: w.lourenzani@unesp.br, Orcid: https://orcid.org/0000-0002-0408-9108

Eduardo Guilherme Satolo: Pós-doutor pelo Programa de Pós-Graduação em Engenharia de Produção e Sistemas Computacionais (MESC-ICT) da Universidade Federal Fluminense (UFF). Doutor, mestre e graduado em Engenharia de Produção pela Universidade Metodista de Piracicaba (UNIMEP). Livre-docente em Administração da Produção pela Universidade Estadual Paulista (UNESP). Professor associado da Faculdade de Ciências e Engenharia da UNESP. Docente permanente do Programa de Pós-Graduação em Agronegócio e Desenvolvimento (PGAD). Pesquisador do Centro de Pesquisa em Administração e Agronegócio (CEPEAGRO/UNESP). E-mail: eduardo.satolo@unesp.br, Orcid: https://orcid.org/0000-0002-8176-2423

Marcellus Marques Caldas: Estágio Pós-Doutoral na Harvard University. Doutor em Economia Aplicada pela Escola Superior de Agricultura "Luiz de Queiroz" da Universidade de São Paulo 
(ESALQ/USP) e em Geografia pela Michigan State University (MSU). Mestre em Economia Aplicada pela Universidade Federal de Viçosa (UFV). Graduado em Economia pela Pontifícia Universidade Católica (PUC). Professor titular do Departamento de Geografia da Kansas State University e diretor do International Research and Faculty Collaboration in the Office of International Programs (OIP).

E-mail: caldasma@ksu.edu, Orcid: https://orcid.org/0000-0003-3086-7054 